\section{MS41-P3 Self-checking bond restraints for Rietveld refinement}

Ivan S. Bushmarinov ${ }^{1}$, Artem O. Dmitrienko ${ }^{1}$

1. A.N. Nesmeyanov Institute of Organoelement Compounds RAS

email: ib@xrlab.ru

The current development of structure determination from powder diffraction (SDPD) approaches has made such structures relatively accessible, with up to 300 Rietveld refined small-molecule structures published each year. However, unlike single-crystal XRD, the R-values for powder XRD data are highly unreliable [1], and the e.s.d. values of structural parameters are well-known [2] to be misleading. In addition, typical Rietveld refinements are heavily restrained, making it difficult to estimate the precision of the resulting structural parameters.

Here, we present a method for checking the validity and accuracy of a Rietveld refined structure requiring only the ability to change the individual values and the overall weight of the restraints. Previously we have found that the use of a custom bond restraint equation [3] in TOPAS helps identifying the "outlying" bonds (more deviating from the restraint value than others). The presence of such "outliers" indicates major errors in structural model. We have successfully applied this approach to structures ranging from organics to uranyl complexes.

In the current study, we consider a statistical model of restraint behavior to tailor the boxplot method for the specific task of outlier detection among bond length deviations. We designed this new method to utilize only classic parabolic restraints readily available in all refinement codes.

We propose an automated refinement protocol providing both initial detection of model errors and a self-check for the final structure. During the latter procedure, each restraint value is varied until it starts producing outliers. The average half-width (AHW) of the obtained restraint ranges represents the absolute extent of bond distortion tolerated by the particular combination of restraints, refinement strategy and powder pattern (typically $\approx 0.1 \AA$, but reaching $0.03 \AA$ for high-quality lab data, according to a small international round robin).

We utilize the developed protocol to study and verify structures inaccessible to both SC-XRD and periodic DFT calculations, in particular Co complexes with strong spin-crossover and oligosiloxanes exhibiting full-molecule disorder.

This research has been supported by the Russian Presidential grant MK-7267.2015.3.

[1] C. Buchsbaum, M.U. Schmidt, Acta Cryst. Sect. B 63 (2007) 926.

[2] R.J. Hill, I.C. Madsen, J. Appl. Cryst. 19 (1986) 10.

[3] I.S. Bushmarinov, A.O. Dmitrienko, A.A. Korlyukov, M.Y. Antipin, J. Appl. Cryst. 45 (2012) 1187.

\section{MS41-P4 Humidity induced phase transitions of hewlysozyme investigated by microcrystalline powder diffraction on a laboratory system}

Detlef Beckers ${ }^{1}$, T. Degen ${ }^{1}$, G. Nénert ${ }^{1}$, S. Saslis ${ }^{2}$, S. Logotheti ${ }^{2}$, F. Karavassili ${ }^{2}$, A. Valmas ${ }^{2}$, I. Margiolaki ${ }^{2}$

1. PANalytical B.V., Almelo, The Netherlands

2. University of Patras, Greece

email: detlef.beckers@panalytical.com

Proteins often crystallize in microcrystalline precipitates. The protein molecules are then surrounded by solvent and their packing arrangement is retained by limited intermolecular contacts. A change in the crystal environment first affects the bulk solvent that fills the intermolecular space, with resulting changes in the crystal structure. In literature it is reported that protein crystals in controlled humidity environments show a large change in unit-cell parameters when the humidity is decreased [1-2]. When a protein crystal is carefully dehydrated, it is in a metastable state in which the crystal initially still retains the original packing structure [2]. Further dehydration may cause the collapse of the crystal lattice: the crystal no longer maintains its packing structure because of the loss of a large amount of bulk solvent. However in some crystals, the dehydration induces a molecular arrangement change resulting in a new crystal structure. This has been already reported for hen egg-white (HEW) lysozyme [3]. While dehydration can induce structural changes, this is also likely to happen upon hydration of the same crystals.

Here, we present our results of microcrystalline protein samples on a laboratory X-ray powder diffractometer including in situ measurements under variable relative humidity conditions. The observed gradual structural changes as well as phase transitions upon dehydration and hydration of HEW lysozyme are analyzed in the relative humidity range $50 \%-95 \%$. We discuss and compare our results with related literature which is so far based on synchrotron studies only.

[1] Kiefersauer, R., et al. (2000). J. Appl. Cryst. 33, 1223-1230.

[2] Dobrianov, I., et al.(1999). J. Cryst. Growth, 196, 511-523.

[3] K. Harata, T. Akiba, Acta Cryst. (2007). D63, $1016-1021$

Keywords: Protein powder diffraction, humidity, laboratory system 